\title{
Balancing cyclic $R$-ary Gray codes II
}

\author{
Mary Flahive \\ Department of Mathematics \\ Oregon State University, \\ Corvallis, OR 97331, USA \\ flahive@math. oregonstate.edu
}

Submitted: Jan 15, 2008; Accepted: Oct 3, 2008; Published: Oct 13, 2008

Mathematics Subject Classification: 05A99,05C45,68R10

\begin{abstract}
New cyclic $n$-digit Gray codes are constructed over $\{0,1, \ldots, R-1\}$ for all $R \geq 2, n \geq 3$. These codes have the property that the distribution of digit changes (transition counts) between two successive elements is close to uniform. For $R=2$, the construction and proof are simpler than earlier balanced cyclic binary Gray codes. For $R \geq 3$ and $n \geq 2$, every transition count is within 2 of the average $R^{n} / n$. For even $R>2$, the codes are as close to uniform as possible, except when there are two anomalous transition counts for $R \equiv 2(\bmod 4)$ and $R^{n}$ is divisible by $n$.
\end{abstract}

\section{Introduction}

For fixed integers $R, n \geq 2$, an $n$-digit $R$-ary Gray code is an ordering of all $n$-strings with digits from $\{0,1, \ldots,(R-1)\}$ such that any two consecutive strings differ in only one digit and that difference is \pm 1 . When the last and first strings also satisfy this property, the code is called cyclic. For example,

$$
20,21,22,12,02,01,11,10,00
$$

is a cyclic 2-digit ternary Gray code. We only consider cyclic codes here.

In the mid-twentieth century Frank Gray [7] designed the eponymous Binary Reflected Gray Code to facilitate relaying information through many repeaters. His code can be generalized to $R \geq 3$, and is cyclic when $R$ is even. The term "Gray code" is now often used to describe listings of combinatorial objects in which successive elements differ in some prescribed minimal way (cf. [11]), but here the term is not used in that general sense.

The transition sequence of a cyclic Gray code records the succession of digit changes $d_{1}, \ldots, d_{R^{n}}$ in the code; the number of times a digit occurs in the transition sequence is 
called its transition count; and the collection of transition counts is the transition spectrum of the code. For example, the transition spectrum of the ternary code in (1.1) is $(5,4)$.

For each pair $R, n$ there are many Gray codes. An important distinguishing characteristic among them is the relative uniformity of transition spectrum. Applications of relatively uniform Gray codes are given in $[2,11]$. For instance, in many present-day parallel machines two processor nodes $A=\left(a_{1} \ldots a_{n}\right)$ and $B=\left(b_{1} \ldots b_{n}\right)$ are connected if and only if they are consecutive elements in a Gray code, and the relative balance of communication loads among different configurations is measured by the relative uniformity of the transition spectrum of the underlying Gray code.

The fact that the code is cyclic implies the sum of all digit changes in any fixed digitposition is congruent to $0(\bmod R)$. Since in a Gray code the possible digit changes are $0, \pm 1$, for even $R$ every transition count must therefore be even. Several authors, among them $[1,2,8,10,14]$, constructed balanced cyclic $n$-digit binary Gray codes in which the difference of any pair of transition counts is at most 2. Exponentially balanced binary codes have also been studied $[13,15]$ where the transition spectrum either has one element or consists of two consecutive powers of 2. Non-binary Gray codes have been considered, for example $[4,3,5,6,8,9,12,14]$.

In [10] John Robinson and Martin Cohn give a method for producing balanced $(n+2)$ digit binary codes from $n$-digit ones. The procedure relies on a subsequence of the original transition sequence that satisfies certain properties, and Girish Bhat and Carla Savage's later proof [2] of the existence of the subsequence was completed by Kiran Kedlaya. More recently it has been noted that the construction (and proof) had already appeared in [1] where it is attributed to T. Bakos. A comment by a referee for our earlier paper [6] motivated us to return to the binary case, and in Section 2 we construct simpler balanced binary codes. More importantly, our proof is much simpler since to obtain the $(n+2)$-digit codes the proof relies only on transition count information from the $n$-digit case.

When all transition counts are equal (the common value must be $R^{n} / n$ ), the code is called uniform or completely balanced. In $[10,16]$ uniform $n$-digit binary codes are constructed for every possible $n$ (a power of 2), but there are few published constructions of uniform codes for arbitrary $R$. For even $R$ and $n$ dividing $R^{n}$, in Theorem 9 we obtain uniform codes when $R \equiv 0(\bmod 4)$ and the construction gives two anomalous transition counts equal to $R^{n} / n \pm 2$ when $R \equiv 2(\bmod 4)$.

When $R^{n}$ is not divisible by $n$, uniform cyclic $n$-digit codes cannot exist and it is reasonable to ask for well-balanced codes in which every transition count is $\left\lfloor R^{n} / n\right\rfloor$ or $\left\lceil R^{n} / n\right\rceil$. In [6] we produced well-balanced 2-digit R-ary Gray codes for every $R \geq 2$ using a natural inductive process in which $(R+2)$-ary codes are constructed from $R$-ary ones. In Section 4 we obtain what we call nearly-uniform codes whose transition counts $T C(1), \ldots, T C(n)$ satisfy

$$
\left|T C(j)-\frac{R^{n}}{n}\right| \leq 2 \text { for all } 1 \leq j \leq n .
$$

(Note that every balanced code is necessarily nearly-uniform.) This continues the work begun in [6], where we constructed codes in which each transition count is within $R-1$ 
of the average. While proofs of previous constructions involved delicate combinatorial arguments, our use of $R^{n} / n$ as a reference point allows simpler proofs based on balancing inequalities.

\section{The Binary Case}

For any $n \geq 2$ and $R \geq 2$, consider the graph whose vertices are all $R$-ary $(n+2)$-strings, in which two vertices are adjacent if and only if they differ in one position by $\pm 1(\bmod R)$. The $(n+2)$-digit $R$-ary Gray codes are the Hamiltonian paths in this Gray graph.

Some binary constructions can be easily described by arranging the vertices of the Gray graph into a $2^{n} \times 4$ rectangular grid in which the rows are labelled by an $n$-digit binary Gray code and the columns numbered consecutively by the cyclic 2-digit code 000111 10. Vertices that are "toroidally adjacent" in this grid are also adjacent in the Gray graph. For instance, the argument in [2] shows there is a choice of 4-digit binary code $a 1, \ldots, a 16$ for which the 6 -digit code graphed in Figure 1 is balanced. (We have labelled the rows in this graph so that the transition digit between $a 15$ and $a 16$ is the one labelled $t_{1}$ in [2].)

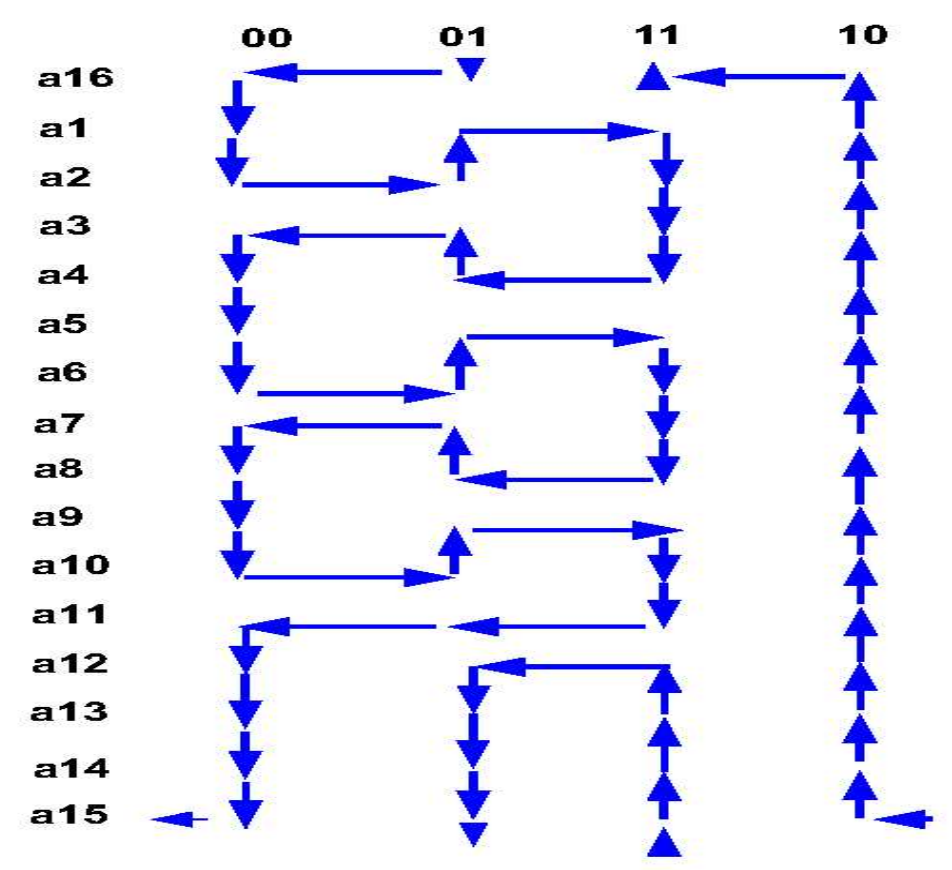

Figure 1: Redrawing the balanced cyclic 6-digit binary code in [2, Figure 1].

For $n=2,3$, Gray's original $n$-digit codes are balanced:

$$
00,01,11,10 \text { and } 000,001,011,010,110,111,101,100 \text {, }
$$


with spectra $(2,2)$ and $(2,2,4)$ respectively. They can be used to obtain the balanced cyclic 4- and 5-digit binary codes graphed in Figure 2. The 4-digit code is uniform, and the 5-digit code has the balanced spectrum $(6,6,8,6,6)$. (Note that denoting the rowindexing $n$-digit code by $a_{1}, \ldots, a_{2^{n}}$, we have used arrow heads to indicate the inclusion of the three edges:

$$
a_{2^{n}} 01 \text { to } a_{1} 01 ; a_{1} 11 \text { to } a_{2^{n}} 11 ; a_{2^{n}} 00 \text { to } a_{2^{n}} 10
$$

corresponding to respective digit changes $1,1, n+1$.)

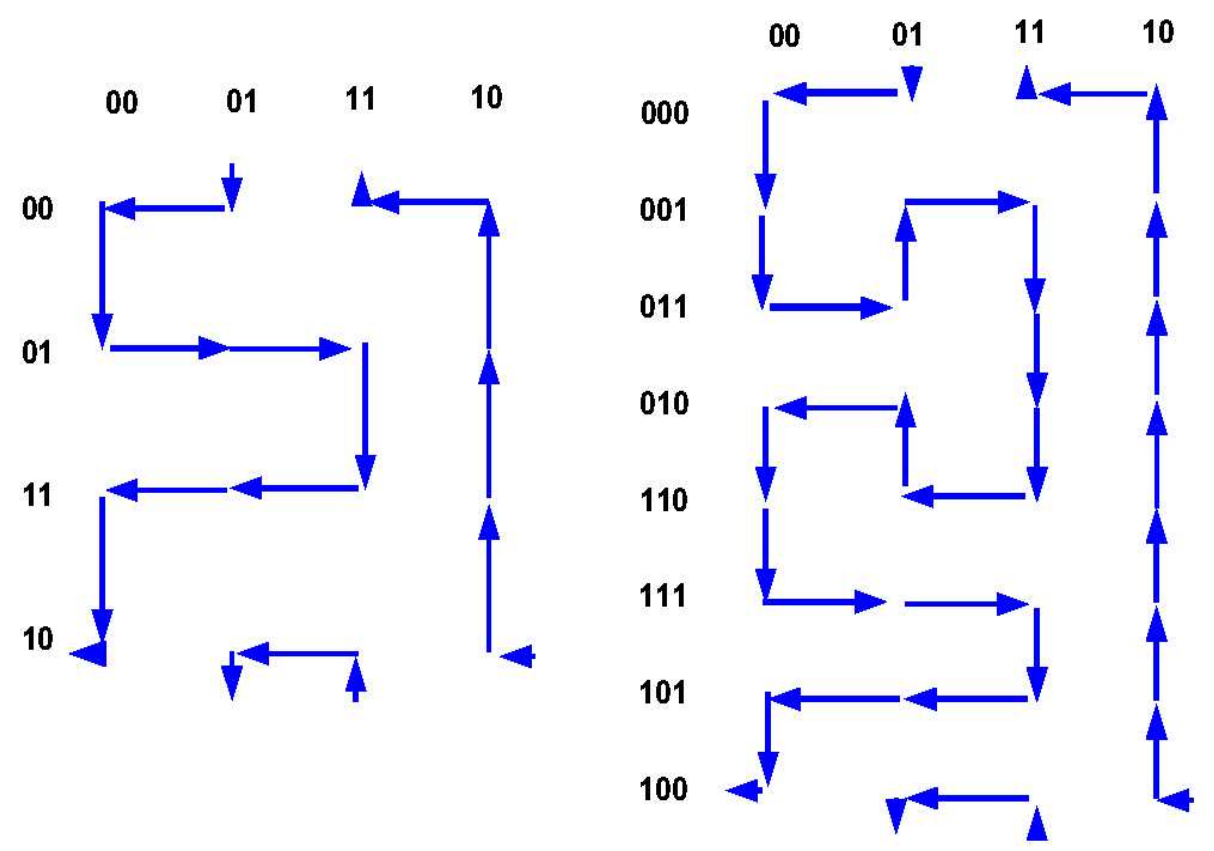

Figure 2: Balanced cyclic 4- and 5-digit binary Gray codes.

The subgraph consisting of the first and last rows of vertices and incident edges is the same in both graphs in Figure 2. Introducing this "notch" yields a uniform spectrum when $2^{n} / n$ is an integer, and the idea of using this notch is inherent in earlier binary constructions. (cf. Figure 1.)

We will construct $(n+2)$-digit balanced codes inductively from $n$-digit ones. For this it is helpful to consider partitions of the $n$-digit code $a_{1}, \ldots, a_{R^{n}}$ into an even number $L \geq 2$ of blocks of the form

$$
\mathcal{B}_{1}:=\left\{a_{1}\right\}, \mathcal{B}_{2}:=\left\{a_{2}, \ldots, a_{i_{2}}\right\}, \ldots, \mathcal{B}_{L-1}:=\left\{a_{i_{L-2}+1}, \ldots, a_{2^{n}-1}\right\}, \mathcal{B}_{L}:=\left\{a_{2^{n}}\right\},
$$

where (as in [6]) the transition digits demarcating the partition blocks are called the connecting digits of the partition and the connecting multiplicity $m_{j}$ refers to the 
number of times the digit $j$ occurs as a connecting digit. For example, in the graphs in Figure 2, the partition blocks $\mathcal{B}_{2}, \ldots \mathcal{B}_{L-1}$ are:

$$
\left\{a_{2}\right\},\left\{a_{3}\right\} \text { and }\left\{a_{2}, a_{3}\right\},\left\{a_{4}, a_{5}\right\},\left\{a_{6}\right\},\left\{a_{7}\right\},
$$

and the connecting digits for the partition are

$$
d_{1}, d_{2}, d_{3}, d_{4} \quad \text { and } \quad d_{1}, d_{3}, d_{5}, d_{6}, d_{7}, d_{8}
$$

with connecting multiplicities

$$
m_{1}=2, m_{2}=2 \quad \text { and } \quad m_{1}=1, m_{2}=1, m_{3}=4 .
$$

Note that $d_{1}, d_{2^{n}-1}, d_{2^{n}}$ are connecting digits in all our partitions.

These partitions are used to describe Hamiltonian paths on the $\left(2^{n}-2\right) \times 3$ rectangular grid obtained by deleting the first and last rows and the last column of the Gray graph in the following way: Beginning with the vertex $a_{2} 00$, traverse all vertices in the first column that belong to the block $B_{2}$, ending with $a_{i_{2}} 00$. After connecting to $a_{i_{2}} 01$ in the second column, traverse (in a backward direction) all vertices in the second column that belong to the block $B_{2}$, and then continue down the third column. This ends at $a_{i_{2}} 11$ which can then be connected to $a_{i_{2}+1} 11$, the first third-column vertex in the third block. This process is continued through the blocks $\mathcal{B}_{3}, \ldots, \mathcal{B}_{L-1}$ of the partition (2.1), yielding a Hamiltonian path on the $\left(2^{n}-2\right) \times 3$ grid which we call the partition path on the grid. It is completed to a Hamiltonian cycle as indicated in the examples in Figure 2. Namely, since $L$ is even, $a_{2^{n}-1} 00$ is always the terminal vertex for the path already constructed. From that vertex, connect to $a_{2^{n}} 00$, over to $a_{2^{n}} 10$ in the last column, up through the last column to $a_{1} 10$ and over to $a_{1} 11$. Consecutively proceeding through

$$
a_{2^{n}} 11, a_{2^{n}} 01, a_{1} 01, a_{1} 00, a_{2} 00
$$

gives a Hamiltonian cycle, which we will call the cycle induced by the partition. Most reported $n$-digit binary codes (for instance, those in $[2,8,10,14,15]$ ) exhibit some partitioning behavior.

Theorem 1. The transition spectrum $(T C(1), \ldots, T C(n+2))$ of the Gray code induced by a partition of an $n$-digit code with transition spectrum $\left(t_{1}, \ldots, t_{n}\right)$ and connecting multiplicities $m_{1}, \ldots, m_{n}$ has transition counts

$$
T C(i)=\left\{\begin{array}{ll}
4 t_{i}-2 m_{i} & \text { if } i \leq n \\
L & \text { if } i>n
\end{array} .\right.
$$

Proof. Scanning across the rows, the (vertical) edges corresponding to a connecting digit are used twice, while the edges corresponding to non-connecting digit changes are used four times. Therefore, each digit $i \leq n$ occurs

$$
2 m_{i}+4\left(t_{i}-m_{i}\right)=4 t_{i}-2 m_{i}
$$

times in the induced Gray code. Further, every block that partitions the rows contains two horizontal edges of the cycle, one corresponding to each of the digits $n+1, n+2$. 
Theorem 1 and its proof is an adaptation of [6, Theorem 3] to the binary case.

Example 2. Here we produce a balanced 6-digit binary code from the uniform 4-digit code in Figure 2. Consider any partition of the 4-digit code in which every connecting multiplicity is 2 , giving $L=8$ blocks. Since each original transition count $t_{i}$ equals 4 , Theorem 1 implies the induced 6-digit code has $T C(i)=12$ for all $i \leq 4$ and $T C(5)=T(6)=L=8$, an unbalanced transition spectrum. If the partition is modified by increasing two of the connecting multiplicities by 1 , say $m_{1}=m_{2}=3$, which is possible since each $t_{i}=4$ ), the number of blocks in the new partition is the even number $L=10$ and the balanced transition spectrum $(10,10,12,12,10,10)$ is obtained. By comparison, the 6-digit code in [2] has a larger notch. (cf. Figure 1.)

Theorem 3. For every $n \geq 2$, there exists a cyclic balanced $n$-digit binary Gray code.

Proof. Note that we've already demonstrated such codes for all $2 \leq n \leq 6$. We will actually prove that for every $n \geq 5$, every cyclic balanced $n$-digit binary Gray code can be partitioned in a way so that the induced $(n+2)$-digit code is balanced.

As in Example 2, the first step is to obtain an initial guess for the connecting multiplicities. For this, for all $i \leq n$ we define the integer $m_{i}$ by

$$
0 \leq 4 t_{i}-\frac{2^{n+2}}{n+2}-2 m_{i}<2
$$

Then $m_{i}$ can be used as a connecting multiplicity for the $i$-th digit if and only if $0 \leq m_{i} \leq$ $t_{i}$. Recalling that our 5 -digit code has transition spectrum $(6,6,8,6,6)$, it can be checked that for this choice $0<m_{i}<t_{i}$ holds for all $i$. For any $n,-2<t_{i}-2^{n} / n<2$ since the original transition spectrum is balanced. It can be checked that this combined with (2.2) gives $0<m_{i}<t_{i}$ for all $n \geq 6$. Therefore, there are (many) partitions that have the positive connecting multiplicities $m_{1}, \ldots, m_{n}$.

In the case when $(n+2)$ divides $2^{n+2}$, since each $4 t_{i}-\frac{2^{n+2}}{n+2}$ is an even integer, the equality on the left-side of (2.2) holds for all $i \leq n$. This implies every $m_{i}$ is even (and so $L$ is even), and $T C(i)=2^{n+2} /(n+2)$ for all $i \leq n$ by Theorem 1. Also,

$$
2^{n+2}=\sum_{i=1}^{n+2} T C(i)=\frac{n}{n+2} 2^{n+2}+2 L
$$

gives $T C(n+1)=T C(n+2)=2^{n+2} /(n+2)$, and the induced code is uniform.

In the remainder of the proof we assume $2^{n+2} /(n+2)$ is not an integer, and each $m_{i}$ defined by

$$
0<4 t_{i}-\frac{2^{n+2}}{n+2}-2 m_{i}<2
$$

If $L$ is odd, the fact that $m_{n}<t_{n}$ allows us to change the partition slightly by increasing the connecting multiplicity of $n$ by 1 , giving a partition with an even number of blocks. 
In any case, by Theorem 1 a partition with these connecting multiplicities induces a cyclic Gray code whose transition counts satisfy

$$
\begin{gathered}
0<T C(i)-\frac{2^{n+2}}{n+2}<2 \text { for all } i<n \quad ; \quad-2<T C(n)-\frac{2^{n+2}}{n+2}<2 ; \\
T C(n+1)=T C(n+2)=L ; \text { and } \sum_{i=1}^{n+2} T C(i)=2^{n+2} \text {. Therefore, } \\
\frac{2^{n+2}}{n+2}-L=\frac{1}{2} \sum_{i=1}^{n}\left(T C(i)-\frac{2^{n+2}}{n+2}\right),
\end{gathered}
$$

which by (2.3) lies in the interval $(-1, n)$, and so $M:=\left\lfloor 2^{n+2} /(n+2)-L\right\rfloor$ satisfies $-1 \leq M<n$. When $-1 \leq M<2$, the code is balanced. For $M \geq 2$, according to whether $M$ is even or odd we increase either all $m_{1}, \ldots, m_{M}$ or all $m_{1}, \ldots, m_{M-1}$ by 1 to get a partition with an even number blocks for which the induced code is balanced.

\section{An Extension of the Binary Construction}

For the remainder of this paper, we consider the question of constructing close-to-uniform cyclic $n$-digit $R$-ary Gray codes for $R \geq 3$. Similar to the binary case, it is helpful to picture the vertices of the $n$-digit $R$-ary Gray graph as arranged in a rectangular grid. Here the columns are labelled as $01 \ldots R-1$ and the rows are indexed by a cyclic $n$-digit Gray code. Setting $\rho$ to be the even integer of $R-2, R-1$, in [6] we used a partition of the rows to construct a Hamiltonian path on the first $\rho+1$ columns of the $R$-ary Gray graph. This allowed us (in [6, Theorem 4]) to obtain $(n+1)$-digit codes in which every transition count is strictly within $\rho$ of the average $R^{n+1} /(n+1)$ (and so is balanced for $R=4)$.

Here we extend the binary construction given in the last section to $R \geq 3$ by considering triples of consecutive columns. This will be used in Section 4 to produce codes in which each transition count is at most 2 from the average. For even $R$, this is a balanced code except when $R \equiv 2(\bmod 4)$ and $R^{n} / n$ is an integer. (cf. Theorem 11.) The spectrum is often less balanced for odd $R$ (Theorem 12).

Earlier work [6, Theorem 4] allows us to assume $R \geq 5$ and $n \geq 3$. Let $q, r$ be a fixed choice of non-negative integers such that $R=3 q+r$. (Note that $q, r$ are not necessarily the usual "quotient" and "remainder"; that is, $r \geq 3$ is possible.) Consider the decomposition of the $R^{n} \times R$ grid into $q$ triplets of three consecutive columns with $r$ residual columns. Within each triplet, the rows will be partitioned using possibly different partitions In each such partition we require that the number of blocks is congruent to $R+1(\bmod 2)$ and in which $\left\{a_{R^{n}}\right\}$ is a block when $R$ is odd. In what follows, such partitions are called permissible. The total number of times a digit $j \leq n$ occurs as a connecting digit in the union of the $q$ partitions will be called its total connecting multiplicity. 

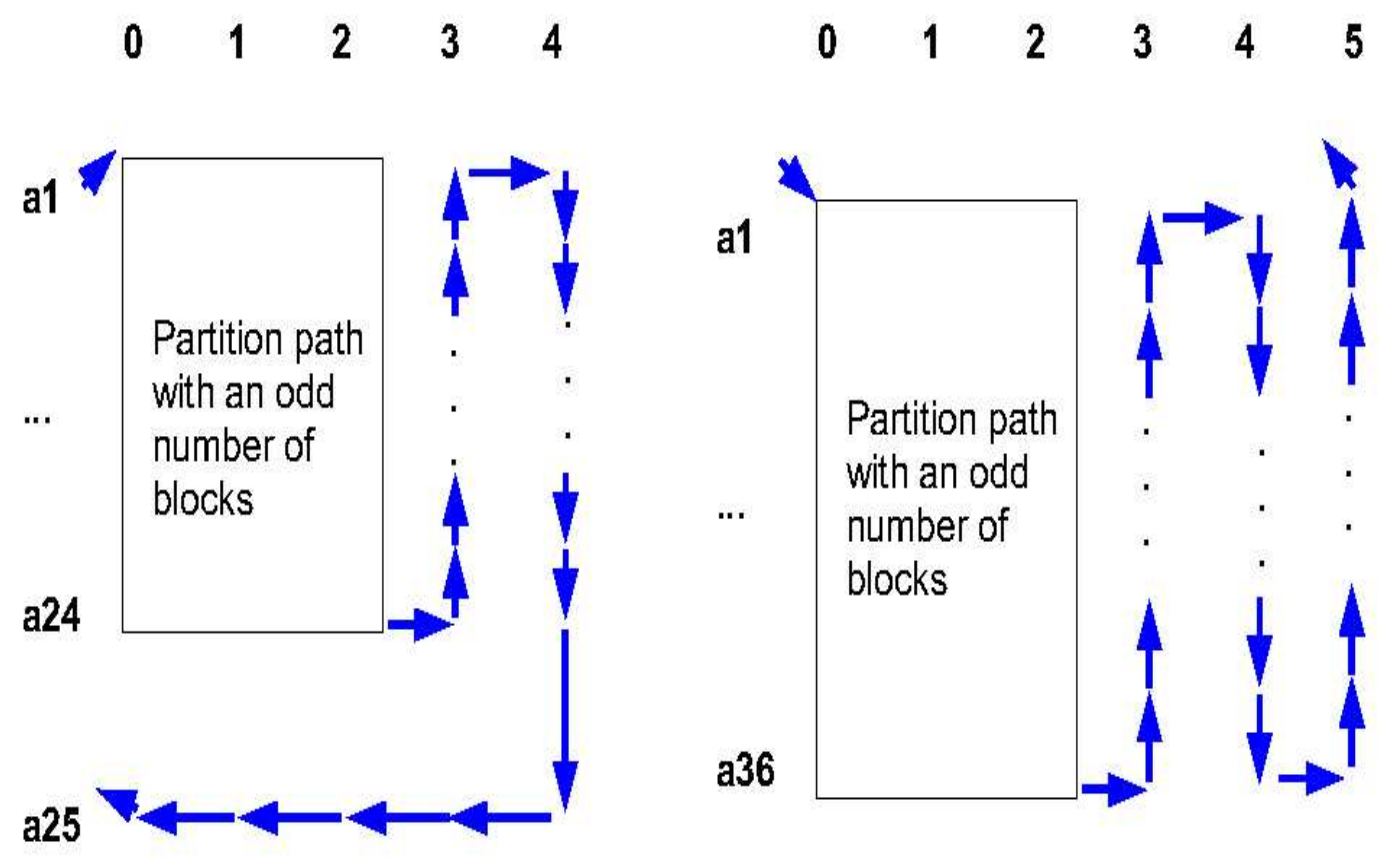

Figure 3: The structure of 3 -digit codes for $R=3 \cdot 1+2$ and $R=3 \cdot 1+3$.

For our description of the construction it's helpful to set $M:=R^{n}-1, R^{n}$, whichever is even. First, on each of the $q$ column triplets use a permissible partition to construct a partition path on the first $M$ rows of the triplet. Since each partition has $R+1(\bmod 2)$ blocks, each of these paths is based on an odd number of partition blocks. The induced path on the first triplet therefore begins with $a_{1} 0$ and ends with $a_{M} 2$, and so can be connected to $a_{M} 3$. Then the (backward) partition path on the second triplet can be traced, ending with the vertex $a_{1} 5$. This process can be continued through all $q$ triplets, creating a Hamiltonian path on the $M \times 3 q$ grid that terminates in either $a_{1}(3 q-1)$ or $a_{M}(3 q-1)$, according to whether $q$ is even or odd. (Here we're using the fact that a triplet contains an odd number of columns.) Figure 3 depicts continuations of the path to Hamiltonian cycles for the decompositions $R=5=3 \cdot 1+2$ and $R=6=3 \cdot 1+3$.

Continuing as in the figure, we extend the path from the $M \times 3 q$ grid to the $M \times R$ grid by going up and down the residual $r$ columns of the $M \times r$ grid in succession. Since $q+r \equiv 3 q+r=R(\bmod 2)$, this ends at either $a_{1}(R-1)$ or $a_{M}(R-1)$ according to whether $R$ is even or odd. When $R$ is even, the terminus $a_{1}(R-1)$ can be connected by a horizontal edge to the initial vertex, and the choice of $M=R^{n}$ means this is a Hamiltonian cycle on the Gray graph. When $R$ is odd, the path ends at $a_{R^{n}-1}(R-1)$. It is completed to a cycle on the Gray graph by connecting to $a_{R^{n}}(R-1)$, traversing the rest of the last row backward, and then connecting to $a_{1} 0$. These cycles will be called the Hamiltonian cycles induced by the decomposition $R=3 q+r$ and the $q$ (permissible) partitions of the $n$-digit code.

This construction depends heavily on the fact that triplets have an odd number of 
columns. A different choice of an odd number of columns would result in different transition counts from those in Theorem 5 . In particular, the summands $2 M_{i}$ would change and result in less ability to balance.

In what follows it is convenient to assume the given $n$-digit code is in standard form, in which its last two transition digits are $d_{R^{n}-1}=2$ and $d_{R^{n}}=1$. This can be accomplished by cycling through the code to get $d_{R^{n}-1} \neq d_{R^{n}}$ and then permuting digits as needed. It is useful to refer to 1 as an eccentric digit, and when $R$ is odd, 2 will also be called eccentric .

Example 4. For the 3-digit codes illustrated in Figure 3, $q=1$ and well-balanced 2digit codes can be used to label the rows, with transition spectra $(12,13)$ for $R=5$ and $(18,18)$ for $R=6$. For $R=5$, any partition with connecting multiplicities $m_{1}=8$, $m_{2}=10$ is permissible, and the 3-digit 5-ary code induced by such a partition has spectrum $(42,43,40)$. Note that the choice of $m_{1}=9, m_{2}=11$ also yields a permissible partition, but the induced code has the less-desirable spectrum $(40,41,44)$. For $R=6$, choosing $m_{1}=16, m_{2}=17$ produces a 6 -ary 3 -digit code with spectrum $(72,74,70)$ that is not uniform. In Theorem 11 we will prove our method cannot construct a uniform 3-digit 6-ary code.

Theorem 5. Let $n \geq 2, R \geq 5$, and let $a_{1}, \ldots, a_{R^{n}}$ be a cyclic $n$-digit $R$-ary Gray code written in standard form with transition spectrum $\left(t_{1}, \ldots, t_{n}\right)$. Then for any decomposition $R=3 q+r$ and any $q$ permissible partitions with total connecting multiplicities $M_{1}, \ldots, M_{n}$, the transition spectrum $(T C(1), \ldots, T C(n+1))$ of the Hamiltonian cycle induced by these partitions has transition counts

$$
T C(i)= \begin{cases}R t_{i}-2 M_{i} & \text { if } i \leq n \text { is non-eccentric } \\ R t_{i}-2 M_{i}-\delta & \text { if } i \text { is eccentric }\end{cases}
$$

where $\delta$ equals either $q+r$ or $q+r-1$ whichever is even.

Proof. In order to calculate the transition counts, we divide the construction of the cycle into three stages: The induced Hamiltonian path on the $M \times 3 q$ grid; the path on the columns of the $M \times r$ grid; and the additional edges that complete the cycle. Note that the $q$ triplets and $r$ residual columns are connected by horizontal edges corresponding to the digit $n+1$ and so are ignored here.

Let $m_{1 j}, \ldots, m_{n j}$ be the connecting multiplicities of the partition in the $j$-th triplet. Within the $j$-th triplet, the transition count of each non-eccentric digit $i \leq n$ is $3 t_{i}-2 m_{i j}$, giving a total of $3 q t_{i}-2 M_{i}$ for the first $3 q$ columns. In each of the remaining $r$ columns, the non-eccentric digit $i$ occurs $t_{i}$ times and so $T C(i)=R t_{i}-2 M_{i}$, as claimed. When $i$ is eccentric, it occurs $t_{i}-1$ times in $a_{1}, \ldots, a_{M}$ and so its transition count in the $M \times R$ grid is

$$
\sum_{j=1}^{q}\left(3\left(t_{i}-1\right)-2\left(m_{i j}-1\right)\right)+\sum_{j=1}^{r}\left(t_{i}-1\right)=R t_{i}-2 M_{i}-q-r .
$$


When $R$ is even, the cycle is completed by adding only horizontal edges; when $R$ is odd, exactly one edge is added for each eccentric digit. Since $q+r \equiv R(\bmod 2)$, this completes the proof.

Corollary 6. For any $R \geq 5$ and $n \geq 3$, the last transition count in the Hamiltonian cycle induced by $R=3 q+r$ and any choice of $q$ permissible partitions of a cyclic $n$-digit $R$-ary Gray code is

$$
T C(n+1)=\left\{\begin{array}{ll}
2 L+2(q+r-1) \equiv 0(\bmod 4) & \text { if } R \text { is odd } \\
2 L+q+r \equiv R(\bmod 4) & \text { if } R \text { is even }
\end{array},\right.
$$

where $L$ is the total number of partition blocks.

Proof. We note the transition spectrum $(T C(1), \ldots, T C(n+1))$ of any cyclic $(n+1)$ digit Gray code satisfies $\sum_{i=1}^{n+1} T C(i)=R^{n+1}$, and that $R=3 q+r \equiv q+r(\bmod 2)$. If $M_{1}, \ldots, M_{n}$ are the total connecting multiplicities then $L=\sum_{i=1}^{n} M_{i}$. Also, by definition of permissible partition,

$$
L \equiv q(R+1)(\bmod 2)
$$

When $R$ is even, $i=1$ is the only eccentric digit, and Theorem 5 implies

$$
\sum_{i=1}^{n} T C(i)=R \sum_{i=1}^{n} t_{i}-2 \sum_{i=1}^{n} M_{i}-\delta=R^{n+1}-2 L-\delta
$$

since the sum of transition counts of an $n$-digit code is $R^{n}$. Then

$$
T C(n+1)=R^{n+1}-\sum_{i=1}^{n} T C(i)=2 L+\delta \equiv 2 q(R+1)+\delta \equiv 3 q+r=R(\bmod 4),
$$

by (3.2). Similarly, for odd $R$,

$$
T C(n+1)=2(L+\delta) \equiv 2(q(R+1)+(q+r-1)) \equiv 0(\bmod 4)
$$

since both $R+1$ and $q+r-1$ are even.

When $R$ is odd, $T C(n+1)$ is always divisible by 4 and so the construction can never construct uniform codes. When $R$ is even and $R^{n+1} /(n+1)$ is an integer, it is always divisible by 4 and so the induced codes cannot be uniform for $R \equiv 2(\bmod 4)$. This is an artifact of the construction that is independent of the chosen decomposition $R=3 q+r$. We return to these observations in Theorems 11 and 12.

\section{Nearly-uniform codes}

In what follows, we will use the decomposition $R=3 q+r$ with $0 \leq r<3$ exclusively. As in the proof of Theorem 3 for the binary case, the strategy here is to obtain $q$ permissible partitions of nearly-uniform $n$-digit codes for which the $(n+1)$-digit induced code is nearly-uniform. The first step is to identify an initial choice of the total connecting multiplicities. 
Lemma 7. Let $R \geq 5$ with $R=3 q+r$ where $0 \leq r<3$, and $\delta=q+r-1$ or $q+r$, whichever is even. Let $t$ be a transition count of either a well-balanced 2-digit code or a nearly-uniform $n$-digit code when $n \geq 3$. For either $\rho=0$ or $\rho=\delta$, the integer $M$ defined by

$$
0 \leq R t-\frac{R^{n+1}}{n+1}-\rho-2 M<2
$$

satisfies $2 q<M<q(t-1)-1$ provided $(R, n) \neq(5,2)$.

Proof. Since

$$
R t-\frac{R^{n+1}}{n+1}-\rho-2<2 M \leq R t-\frac{R^{n+1}}{n+1}-\rho
$$

it suffices to show that

$$
R t-\frac{R^{n+1}}{n+1}-\rho-2>4 q \text { and } R t-\frac{R^{n+1}}{n+1}-\rho<2 q(t-1)-2 .
$$

The first inequality in (4.1) follows from $\rho+4 q \leq 5 q+r \leq 5 R / 3$ and $t \geq R^{n} / n-2$ :

$$
R t-\frac{R^{n+1}}{n+1}-\rho-2-4 q \geq \frac{R^{3}}{6}-\frac{11 R}{3}-2=\frac{R\left(R^{2}-22\right)-12}{6}>0
$$

by $n \geq 2$ and $R \geq 5$. Since $q \geq(R-2) / 3$ and $\rho \geq 0$, for the second inequality in (4.1) it suffices to prove that

$$
\frac{R^{n+1}}{n+1}-R t+\frac{2(R-2)}{3}(t-1)-2=\frac{R^{n+1}}{n+1}-\frac{2 R+2}{3}-\frac{(R+4) t}{3}>0 .
$$

When $n=2$, then $R \neq 5$, and $t \leq\left(R^{2}+1\right) / 2$ gives

$$
(R+4) t \leq\left(R^{3}+4 R^{2}+R+4\right) / 2 .
$$

Therefore, (4.2) is true since

$$
\frac{R^{3}}{3}-\frac{2 R+2}{3}-\frac{R^{3}+4 R^{2}+R+4}{6}=\frac{R^{3}-4 R^{2}-5 R-8}{6},
$$

a strictly increasing function on $R \geq 6$ that is positive at $R=6$.

When $n \geq 3$,

$$
(R+4) t \leq(R+4)\left(R^{n} / n+2\right)=\left(R^{n+1}+4 R^{n}\right) / n+2 R+8
$$

and so

$$
\frac{R^{n+1}}{n+1}-\frac{2 R+2}{3}-\frac{(R+4) t}{3} \geq \frac{R^{n}}{3 n}\left(R \frac{2 n-1}{n+1}-4\right)-\frac{4 R+10}{3},
$$

which is an increasing function of $n \geq 3$, and at $n=3$ can be checked to be positive for all $R \geq 5$. 
Lemma 8. Let $q, n$ and $t_{1}, \ldots, t_{n}$ be positive integers. If $M_{1}, \ldots, M_{n}$ are integers such that $2 q<M_{i}<q\left(t_{i}-1\right)$ for all $i$, then for any integer a there exists an $n \times q$ integer matrix $\left[m_{i j}\right]$ satisfying all of the following:

(a) $1 \leq m_{i j} \leq t_{i}$, for all $1 \leq i \leq n$ and $1 \leq j \leq q$;

(b) for all $1 \leq i<n$, the row-sum $\sum_{j=1}^{q} m_{i j}$ equals $M_{i}$ and the last row-sum equals either $M_{n}$ or $M_{n}+1$;

(c) for all $j=1, \ldots, q$, the column-sum $\sum_{i=1}^{n} m_{i j} \equiv a(\bmod 2)$.

Proof. For fixed $i$, let $u, v$ be the integers such that $M_{i}=u q+v$ with $0 \leq v<q$, and define

$$
m_{i j}=\left\{\begin{array}{ll}
u+1 & \text { if } 1 \leq j \leq v \\
u & \text { if } v<j \leq q
\end{array} ;\right.
$$

that is, "distribute the $M_{i}$ as uniformly as possible across the columns of the $i$-th row". For this choice of entries in the $i$-th row, $\sum_{j=1}^{q} m_{i j}=M_{i}$ holds, and $2 q<M_{i}<q\left(t_{i}-1\right)$ implies $2 \leq u<t_{i}-1$. Therefore, $2 \leq m_{i j}<t_{i}$ for all $1 \leq j \leq q$ and the matrix $\left[m_{i j}\right]$ has the first two properties.

Consider all columns $j_{1}<\ldots<j_{T}$ for which the column-sum $L_{j}:=\sum_{i=1}^{n} m_{i j}$ is not congruent to $a(\bmod 2)$. For any pair $j_{1}<j_{2}$, we can modify the choices of $m_{n j_{1}}$ and $m_{n j_{2}}$ (both in the last row) by subtracting 1 from $m_{n j_{1}}$ and adding 1 to $m_{n j_{2}}$. This conserves the sum $M_{n}$. Since $2 \leq m_{i j} \leq t_{i}$, property (a) still holds for all new $m_{i j}$ and the parity of both $L_{j_{1}}$ and $L_{j_{2}}$ has been changed to $a(\bmod 2)$. Since pairs of pathological $L_{j}$ can be modified in this way, it remains to consider odd $T$. In that case, increasing $m_{n j_{T}}$ by 1 increases the row-sum $M_{n}$ by 1 , and changes the congruence class of $L_{j_{T}}(\bmod 2)$ to $a$ $(\bmod 2)$.

Theorem 9. For each $R \geq 3, n \geq 1$ there exists a nearly-uniform cyclic $(n+1)$-digit $R$-ary Gray code. In particular, its transition counts $T C(1), \ldots, T C(n+1)$ satisfy

$$
-2 \leq T C(j)-\frac{R^{n+1}}{n+1}<2 \text { for all } j \leq n \quad \text { and }-2<T C(n+1)-\frac{R^{n+1}}{n+1} \leq 2 .
$$

Proof. For fixed $R$, the proof is by induction on $n \geq 2$. By Theorem 1 in [6], there exists a well-balanced 2-digit code for all $R \geq 2$. As mentioned earlier, Theorem 4 in [6] allows us to assume $R \geq 5$. Since a nearly-uniform cyclic 3 -digit 5 -ary code was constructed in Example 4, we may also assume $(R, n) \neq(5,2)$.

Let $q, r$ be the integers with $R=3 q+r$ and $0 \leq r<3$, and let $a_{1}, \ldots, a_{R^{n}}$ be any nearly-uniform $n$-digit code in standard form with transition spectrum $\left(t_{1}, \ldots, t_{n}\right)$, which is assumed to be well-balanced when $n=2$. For $1 \leq i \leq n$, define $\rho_{i}=0$ when $i$ is a non-eccentric digit and $\rho_{i}=\delta$ when $i$ is eccentric. Since $(R, n) \neq(5,2)$ and each pair $t_{i}, \rho_{i}$ satisfies the hypothesis of Lemma 7 , there exist integers $k_{1}, \ldots, k_{n}$ such that for all $i \leq n$

$$
2 q<k_{i}<q\left(t_{i}-1\right)-1
$$

and $T_{i}:=R t_{i}-\rho_{i}-2 k_{i}$ satisfies

$$
0 \leq T_{i}-\frac{R^{n+1}}{n+1}<2
$$


Defining $M:=\left\lfloor\sum_{j=1}^{n}\left(T_{j}-\frac{R^{n+1}}{n+1}\right) / 2\right\rfloor$,

$$
2 M \leq \sum_{j=1}^{n}\left(T_{j}-\frac{R^{n+1}}{n+1}\right)<2 M+2
$$

and (4.5) implies $0 \leq M<n$. Setting $M^{*}$ to be either $M$ or $M+1$, whichever satisfies

$$
\sum_{i=1}^{n} k_{i}+M^{*} \equiv q(R+1)(\bmod 2),
$$

then $0 \leq M^{*} \leq M+1 \leq n$ allows us to define the integers $M_{1}, \ldots, M_{n}$ by

$$
M_{i}= \begin{cases}k_{i}+1 & \text { if } 1 \leq i \leq M^{*} \\ k_{i} & \text { if } M^{*}<i \leq n\end{cases}
$$

where

$$
\sum_{i=1}^{n} M_{i} \equiv q(R+1)(\bmod 2)
$$

by $(4.7)$, and $2 q<M_{i}<q\left(t_{i}-1\right)$ for all $i$ by (4.4).

For $a:=q(R+1)$, let $\left[m_{i j}\right]$ be a matrix that satisfies the conclusions of Lemma 8 . Property (a) implies for each $j$ there exist partitions of the $n$-digit code with (positive) connecting multiplicities $m_{1 j}, \ldots, m_{n j}$. Since every digit is chosen at least once as a connecting digit, when $R$ is odd we can restrict ourselves to partitions in which $\left\{a_{R^{n}}\right\}$ is a partition block. Property (c) therefore implies every such partition is permissible. By property (b), $M_{i}$ is the row-sum for all $i<n$; that is, $M_{1}, \ldots, M_{n-1}$ are the total connecting multiplicities for the first $n-1$ digits. Finally, by property (c) the sum of the column-sums is congruent to $q(R+1)(\bmod 2)$, and so $(4.8)$ then implies the last row-sum cannot be $M_{n}+1$. Therefore, $M_{1}, \ldots, M_{n}$ are the total connecting multiplicities of the $q$ permissible partitions and Theorem 5 can be applied.

The induced cyclic $(n+1)$-digit Gray code therefore has transition counts

$$
T C(i)=\left\{\begin{array}{ll}
T_{i}-2 & \text { if } i \leq M^{*} \\
T_{i} & \text { if } M^{*}<i \leq n
\end{array},\right.
$$

and by (4.5)

$$
-2 \leq T C(i)-\frac{R^{n+1}}{n+1}<0 \text { for } i \leq M^{*} \quad \text { and } \quad 0 \leq T C(i)-\frac{R^{n+1}}{n+1}<2 \text { for } M^{*}<i \leq n .
$$

As for the last transition count, remember $\sum_{i=1}^{n+1} T C(i)=R^{n+1}$ and so

$$
\frac{R^{n+1}}{n+1}-T C(n+1)=\sum_{i=1}^{n}\left(T C(i)-\frac{R^{n+1}}{n+1}\right)=\sum_{i=1}^{n}\left(T_{i}-\frac{R^{n+1}}{n+1}\right)-2 M^{*} \text {. }
$$


By (4.6) the choice of $M^{*}=M, M+1$ therefore gives

$$
-2 \leq 2\left(M-M^{*}\right) \leq \frac{R^{n+1}}{n+1}-T C(n+1)<2\left(M-M^{*}\right)+2 \leq 2,
$$

proving the induced code is nearly-uniform.

Example 10. We use the method in the proof of Theorem 9 to construct nearly-uniform 3-digit Gray codes for $R=6,8$ and well-balanced 4-digit codes for $R=5$.

When $R$ is even, $i=1$ is the only eccentric digit and so for $n=2$,

$$
T_{1}=R t_{1}-(q+r)-2 k_{1} \text { and } \quad T_{2}=R t_{2}-2 k_{2},
$$

both even integers. Also, by (4.5),

$$
\frac{R^{3}}{3} \leq T_{i}<\frac{R^{3}}{3}+2 .
$$

For $R=8$, we begin with a uniform 2-digit code with transition counts $t_{1}=t_{2}=32$. Since $T_{1}, T_{2}$ are even, $T_{1}=T_{2}=172$ by (4.10), and $R=2 \cdot 3+2$ gives $q+r=4$. Solving (4.9) yields $k_{1}=40 ; k_{2}=k_{1}+(q+r) / 2=42$, and $k_{1}+k_{2}$ is even as required. However, the residual $8^{3}-344=168$ is not within 2 of the average $R^{3} / 3$. In order to balance while maintaining the evenness of $k_{1}+k_{2}$, each of the original $k_{1}, k_{2}$ must be increased by 1 , giving total multiplicities 41,43 . Lemma 7 can now be used to find the actual code: For instance, since $t_{1}=t_{2}=32$, multiplicities $m_{11}=m_{21}=32$ could be chosen for the partition on the first column triplet, leaving $m_{12}=41-32=9$ and $m_{22}=43-32=11$ for the second.

When $R=6$, (4.10) implies $T_{1}=T_{2}=72$. Since $q+r=2$ then $k_{1}=k_{2}+1$, giving an odd sum. Therefore, one of the $k_{i}$ must be increased, resulting in the nearly-uniform spectrum $(70,72,74)$. (For instance, $k_{1}=17, k_{2}=19$ can be used.) In Theorem 11, we prove our construction cannot yield a uniform 3-digit code for $R=6$.

Finally, we show the 3-digit 5-ary code in Example 4 can be used to construct a wellbalanced 4-digit one. Since $\left\lfloor 5^{4} / 4\right\rfloor=156$ then Corollary 6 combined with (4.5) implies the codes constructed by our method must have $T C(4)=156$. It therefore suffices to show there exist total multiplicities $k_{1}, k_{2}, k_{3}$ such that $k_{1}+k_{2}+k_{3}$ is even and

$$
156=T_{1}=5 t_{1}-2-2 k_{1}, 157=T_{2}=5 t_{2}-2-2 k_{2}, 156=T_{3}=5 t_{3}-2 k_{3}
$$

where $t_{1}=42, t_{2}=43, t_{3}=40$, from Example 4 . Considering each of these equations modulo 4 , it can be seen that each $k_{i}$ is even and so their sum is even. (In fact, $k_{1}=$ $26, k_{2}=28, k_{3}=22$.)

Theorem 11. Let $n \geq 3$ and $R \geq 4$ be even.

(a) If $n$ does not divide $R^{n}$, our construction yields a balanced cyclic n-digit $R$-ary Gray code.

(b) If $n$ divides $R^{n}$, our construction yields a uniform cyclic $n$-digit $R$-ary Gray code when $R \equiv 0(\bmod 4)$. When $R \equiv 2(\bmod 4)$ the construction yields a code in which all but two transition counts equal $R^{n} / n$. 
Proof. Every transition count is even, and by definition of a nearly-uniform code, every transition count lies in the interval $\left[R^{n} / n-2, R^{n} / n+2\right]$. When $R^{n} / n$ is not an integer, the interval contains only two even integers and so the code must be balanced

When $R^{n} / n$ is an integer, it is divisible by 4 . Since $T C(n+1) \equiv R(\bmod 4)$ by Corollary 6, then (4.3) implies

$$
T C(n)=\left\{\begin{array}{ll}
R^{n} / n & \text { if } R \equiv 0(\bmod 4) \\
R^{n} / n+2 & \text { if } R \equiv 2(\bmod 4)
\end{array} .\right.
$$

Also, for $i \leq n$, from (4.3) we see that for $i<n$ the only possibilities for $T C(i)$ are $R^{n} / n$ and $R^{n} / n-2$, and the result follows from the fact that the sum of the transition counts is $R^{n}$.

In Corollary 6 it was proved that for odd $R$ the last transition count in the induced codes is always divisible by 4 . Because of this, the codes are never uniform but the next result shows our construction admits further uniformization.

Theorem 12. If $R \geq 5$ is odd and $n \geq 3$ is such that $R^{n} / n$ is an integer then the construction can be used to obtain a balanced code.

Proof. From the proofs of Lemma 8 and Theorem 9, there is a nearly-uniform cyclic $n$-digit $R$-ary code in which at least one column triplet has a partition with all connecting multiplicities satisfying $1 \leq m_{i j}<t_{i}$, and so every connecting multiplicity in that partition can be changed by \pm 1 . Therefore, if $i, k<n$ are digits with $T C(i)<R^{n} / n<T C(k)$, there exists another induced code in which the transition count for $i$ is $T C(i)+2$, the count for $j$ is $T C(j)-2$, and all other transition counts are unchanged. We use this "pair balancing" in what follows.

Let $a:=R^{n} / n$. Since $a$ is an odd integer, Corollary 6 implies $T C(n)=a \pm 1$. Therefore, by way of contradiction we may assume there exists at least one digit $i<n$ such that $T C(i) \notin\{a, a \pm 1\}$. We may assume $i=n-1$. If $T C(n-1)=a-2$ then

$$
0=\sum_{i=1}^{n}(T C(i)-a)=\sum_{i=1}^{n-2}(T C(i)-a)-2 \pm 1
$$

and there exists $j \leq n-2$ such that $T C(j) \in\{a+1, a+2\}$. Using pair balancing on $T C(j)$ and $T C(n-1)$ results in an induced $n$-digit code in which $T C(n-1)=a$ and $T C(j) \in\{a-1, a\}$. A similar argument works for $T C(n-1)=a+2$, and can be continued to obtain a code in which all transition counts are in the set $\{a, a \pm 1\}$. 


\section{References}

[1] A. Ádám. Truth functions and the problem of their realization by two-terminal graphs. Akadémiai Kiadó, Budapest, 1968. pages 28-37.

[2] Girish S. Bhat and Carla D. Savage. Balanced Gray codes. Electronic Journal of Combinatorics, 3(1):Research Paper 25, 11 pp. (electronic), 1996.

[3] B. Bose and B. Broeg. Lee distance Gray codes. In Proceedings of the International Symposium on Information Theory, 1995.

[4] B. Bose, B. Broeg, Y. Kwon, and Y. Ashir. Lee distance and topological properties of $k$-ary $n$-cubes. IEEE Transactions on Computers, 44:1021-1030, 1995.

[5] Martin Cohn. Affine m-ary Gray codes. Information and Control, 6:70-78, 1963.

[6] Mary Flahive and Bella Bose. Balancing cyclic R-ary Gray codes. Electronic Journal of Combinatorics, 14(1):Research Paper 31, 13 pp. (electronic), 2007.

[7] W. M. Goodall. Television by pulse code modulation. Bell System Tech. J., pages 33-45, 1951.

[8] Donald E. Knuth. The Art of Computer Programming, volume 4, Fascicle 2. AddisonWesley, 2005.

[9] Chi-Jen Lu and Shi-Chun Tsai. A note on iterating an $\alpha$-ary Gray code. SIAM J. Discrete Math., 14(2):237-239 (electronic), 2001.

[10] John P. Robinson and Martin Cohn. Counting sequences. IEEE Transactions on Computers, 30(1):17-23, 1981.

[11] Carla Savage. A survey of combinatorial Gray codes. SIAM Rev., 39(4):605-629, 1997.

[12] Bhu Dev Sharma and Ravinder Kumar Khanna. On m-ary Gray codes. Inform. Sci., 15(1):31-43, 1978.

[13] I Nengah Suparta. A simple proof for the existence of exponentially balanced Gray codes. Electron. J. Combin., 12:Note 19, 5 pp. (electronic), 2005.

[14] I Nengah Suparta. Counting sequences, Gray codes and Lexicodes. PhD thesis, Delft University of Technology, 2006.

[15] A. J. van Zanten and I. N. Suparta. Totally balanced and exponentially balanced Gray codes. Discrete Analysis and Operations Research, Ser. I, 11:81-98, 2004.

[16] David G. Wagner and Julian West. Construction of uniform Gray codes. Congr. Numer., 80:217-223, 1991. 